\title{
Strandgut, Treibgut
}

\author{
Michael Niehaus
}

\begin{abstract}
This article imagines the sea, through a focus on the coast, as a space of remembrance. The coast is a zone where the sea, understood as a storage medium, deposits natural things and artefacts in an uncontrolled way. The essay distinguishes two historical phases: In the first phase - up until the 19th century - the commercial value of the deposited things is the central concern. Accordingly, the beach appears first and foremost as a chaotic zone of (collective) beach robbery. In the second phase, an aesthetic and contemplative relationship between man and the beach can take hold, which manifests itself particularly in the (solitary) beach walk. Things are assigned a symbolic value and memorial character, making the sea a special reservoir of memory. Understood in this framework, the contemporary sea, predominantly depositing sea garbage, creates less an image of transculturality than of the inescapable globalisation of Western civilization.
\end{abstract}

Title: Flotsam and Jetsam

Keywords: beach theft; storage memory; found objects; commemorative mark; globalization

\section{Von der Küste zUm Strand}

Das Meer ist nicht grenzenlos; es wird von Küsten begrenzt, von Stränden. Im Normalfall sehen wir das Meer von der Küste aus. Wenn wir von der Küste als Küste sprechen, denken wir sie als die Zone des Austauschs, des Übergangs, der Schwelle, während das Meer für uns ein Raum für sich ist. In seiner Studie Meereslust. Das Abendland und die Entdeckung der Küste 1750-1840 hat Alain Corbin die Entwicklung nachgezeichnet, die unsere abendländische Wahrnehmung der Meere von der Küste genommen hat, bevor es, wie er sich ausdrückt, zur »Erfindung des Strandes« (Corbin 1990: 319) gekommen ist, wie wir ihn kennen. Der Strand als ein friedlicher Aufenthaltsort für den Menschen ist nicht nur deshalb voraussetzungsreich, weil das Baden im Meer eine späte Errungenschaft ist. Das Meeresbaden - ob zum Vergnügen oder der Gesundheit halber - stellt nur ein Element des Ensembles von Praktiken dar, die mit den zu gehegten Stränden gewordenen Küstenstreifen verbunden sind. Corbin macht in diesem Zusammenhang den Begriff des otium - der Muße - stark. Bereits von den vornehmen Römern der Antike konnte eine Villa am Meer (in sicherer Entfernung von ihm) als ein Raum der Entspannung und der Kontemplation genossen werden (vgl. ebd.: 320-322); in den Texten zur Ästhetik aus dem 18. Jahrhundert ist der Anblick des Meeres (aus einer Halbdistanz) eines der topischen Beispiele für das 
Erhabene; und in anderer Weise ist auch das gesellige Leben am Strand (in der Nähe des Wassers) mitsamt den dort ausgeübten Tätigkeiten zunächst einmal eine Form des Müßiggangs, wodurch eine Opposition zu jenen Teilen der Bevölkerung entsteht, deren unmittelbare Begegnung mit dem Meer sich ihrem Arbeitsleben verdankt.

Den Strand als geselligen Natur-Kultur-Raum der Freizeitbeschäftigungen den Strand, den man kultursemiotisch »als Text lesen« (Fiske 2000: 56 ) kann gibt es nur, weil das Meer in besonderer Weise dazu in Lage ist, ein vereinzeltes Subjekt in eine im weitesten Sinne ästhetische Stimmung von unterschiedlicher $>$ Tiefe $<$ zu versetzen. Diese Tiefe wurde erstmals in der Romantik ausgelotet, wobei die Romantiker freilich, wie Corbin beschreibt, »viel von früheren Modellen« (Corbin 1990: 213) übernehmen. Das Meeresufer wird »zum privilegierten Ort der Selbstentdeckung«, der Strand zum Auslöser einer »besondere[n] Schwingung des Ich«, und der »Traum von einer Verschmelzung mit den elementaren Kräften« kann sich hier ebenso entfalten wie das »Phantasma des Verschlungenwerdens«; überdies lässt die »Leere des Ozeans, zum metaphorischen Ort des persönlichen Schicksals erhoben, [...] den Strand als einen Grenzbereich erscheinen, der den Spaziergänger [...] zu einer periodischen Lebensbilanz auffordert.« (Ebd.: 214) Emblematisch vor Augen geführt wird dies in dem revolutionären (und entsprechend schnell berühmt gewordenen) Gemälde Mönch am Meer (1809) von Caspar David Friedrich, in dem der gemalte Raum zu einem »inneren Raum« (ebd.: 217) für ein einsames Subjekt geworden ist. Das Individuum der Romantik, so fasst Corbin zusammen, komme nicht mehr an die Küste, um »die Grenzen zu bewundern, die Gott der Gewalt der Meere gesetzt hat«, sondern hoffe, auf »der Suche nach sich selbst, [...] seine eigenen Grenzen zu entdecken oder, besser gesagt, sich wiederzufinden « (ebd.: 214). Wenn das Subjekt in dieser Weise müßig ist, wird das Meer zu einem abstrakten Gegenüber, einem Imaginären und einem Ort der Imagination. Man könnte sagen, es kommt zu keiner konkreten Verbindung. Man kann dem Meer auch - von der Küste aus etwas Konkretes übergeben, vor allem aber kann man vom Meer etwas vor die Füße gespült bekommen. Dann wird die Verbindung konkret. Der Art und Weise, in der das Meer über Dinge mit uns in Verbindung tritt, widmen sich die folgenden Überlegungen.

\section{StrandRAUB}

Das Meer lässt sich - wie vieles andere auch - als ein Speichermedium auffassen, das freilich nicht Informationen, sondern Dinge gespeichert hat. Den Output dieses Speichers findet man in der Zone des Strandes, als angeschwemmtes oder zu bergendes Strandgut. Das ist natürlich eine sehr spezielle Betrachtungsweise. Sie lenkt aber den Blick darauf, dass das Meer als ein Speichermedium ein Gegenüber und ein Akteur ist, der - gleichsam - etwas Konkretes hergibt. Was das Meer hergibt, ist insofern ein Gut, als es unter dem Wert-Aspekt betrachtet werden kann. 
Zunächst ist daran zu erinnern, dass die Orte, an denen Wertgegenstände einen (neuen) Eigentümer erhalten, stets Gegenstand von Regelungen sind - so etwa, wenn etwas getauscht, erworben oder geschenkt wird: Der Eigentümer ist der rechtmäßige Besitzer. Wer das, was er sein Eigentum nennen darf, nicht selber hergestellt hat, hat es in der Regel von einem Voreigentümer übertragen bekommen. Wenn jemand Dinge am Strand findet und aufliest, ist er noch nicht deren Eigentümer geworden, er hat sich nur besitzlose Sachen angeeignet. Wenn diese Sachen einen Eigentümer gehabt haben, sind sie zunächst einmal nicht herrenlos. Herrenlos im juristischen Sinne sind allerdings das Wasser des Meeres selbst und die Dinge, die es von Natur aus an den Strand spült (Dinge, die nie Sachen gewesen sind). Damit eine Sache herrenlos ist, bedarf es eines Eigentumsverzichts (Dereliktion). Haben die vormaligen Eigentümer auf das Eigentum an den Sachen, die man am Strand findet, verzichtet? Das ist nicht gewiss. Nach deutschem Recht fällt das Strandgut unter das Fundrecht, welches für Sachen im Wert von mehr als 10 Euro eine Abgabepflicht festlegt. Erst nach Ablauf eines halben Jahres geht das Eigentum, wenn kein Eigentümer einen Anspruch geltend gemacht hat, an den Finder.

Es ist nicht selbstverständlich, dass das Strandgut unter das Fundrecht fällt. In Deutschland ist das erst seit 1990 der Fall. Diese Subsumtion markiert den (vorläufigen) Endpunkt einer langen Geschichte, in der es darum ging, die umkämpfte Zone des Strandes zu pazifizieren. Hat nicht das, was man am Strand finden kann, das Meer selbst hergegeben? Hat das Meer als Speichermedium nicht alle anderen Eigentumsverhältnisse gewissermaßen gelöscht? Muss der Strand nicht ein Ort besonderen Rechtes sein? Schon in hellenistischer Zeit wurde das, was auf dem Meer und an den Küsten geschieht, Gegenstand rechtlicher Regelungen. Die erste, von der wir wissen, ist die Lex Rhodia de iactu aus dem 3. vorchristlichen Jahrhundert (vgl. Wagner 1997), die später in das Römische Recht eingearbeitet wurde. Sie behandelte in erster Linie den Seewurf, das heißt die diffizilen Fragen des Schadensausgleichs, wenn in Seenot befindliche Schiffe Teile des ihnen von den Eigentümern anvertrauten Frachtguts über Bord werfen müssen, um dem Schiffbruch zu entgehen. Schon das Römische Recht legt aber auch fest, dass eine »zur Entlastung eines Schiffs über Bord geworfene Sache [...] im Eigentum ihres Herrn« verbleibt und eben »nicht dem Ergreifer« gehört, »weil sie nicht als derelinquiert, als herrenlose Sache erachtet wird; denn der Eigentümer hat nicht die Absicht, mentem, sie aufzugeben, vielmehr würde er sie, wenn er sie wiederfände, mitnehmen« (ebd.: 370): Auch derjenige, der seine Sachen über Bord wirft, hofft, dass das Meer als ihr temporärer Speicher (sozusagen als - wenn auch unsicheres -Depot) fungieren wird.

Mit dem Wort Strandrecht wird das ursprünglich überall verbreitete und gleichsam >natürliche - nämlich vom Meer verliehene - Recht der Küstenbewohner bezeichnet, das, was sie am Strand auflesen oder in der Uferzone bergen können, als ihr Eigentum zu betrachten, und häufig auch die als rechtlo- 
se Fremde betrachteten Überlebenden eines Schiffbruchs zu versklaven. ${ }^{1}$ Mit anderen Worten, das Strandrecht läuft darauf hinaus, den Strand als einen gewohnheitsrechtlichen Ort jenseits des kodifizierten Gesetzes zu statuieren (vgl. Wolf 2013: 296f.).

In den Zeiten, in denen die Schifffahrt aufgrund der beschränkten Manövrierfähigkeit und Sturmanfälligkeit der Schiffe, der rudimentären nautischen Instrumente und der Ungenauigkeit der Karten sehr gefahrvoll war, galt dieses Gewohnheitsrecht als eine willkommene zusätzliche Einkommensquelle für diejenigen Küstenbewohner, die nicht das Glück hatten, von einem Hafen zu profitieren. Verbreitet sind die Geschichten, in denen es nicht beim Auflesen und Bergen von Strandgut blieb (vgl. Rath 2007; Wolf 2013: 293-313). Bereits im Römischen Recht wird unter Strafe gestellt, wer Schiffbrüchige daran hindert, sich selbst um die Bergung der Fracht zu kümmern (vgl. Wagner 1997: 377; Engelbrecht 1790: 31-33), oder gar durch Aufstecken irreführender Lichter das Stranden von Schiffen zu provozieren versucht (Wagner 1997: 379). Außerhalb des Römischen Weltreiches, etwa an der Nord- und Ostsee, herrschte das alte Strandrecht noch bis ins 11. Jahrhundert hinein ungebrochen; für ein Schiff war also gewissermaßen »alles feindlich, sobald man den Heimathafen verlassen hatte« (Rath 2007: 60; vgl. auch Wolf 2013: 296). Im Mittelalter setzte sich die Kirche für die Abschaffung des Strandrechtes ein; ein Konzil bestimmte im Jahr 1110, dass, wer Sachen aus einem Schiffbruch raubt und einen Schiffbrüchigen tötet, exkommuniziert werden solle, schränkte dies aber auf Opfer christlicher Religion ein. Auch die deutschen Kaiser bemühten sich um die Abschaffung des Strandrechts (vgl. ebd.). Den Hauptanstoß für dessen Zurückdrängung gaben jedoch die Bemühungen der Hansestädte um Risikoverringerung (vgl. Hansen 2001). Das erste »Privileg einer Strandrechtsbefreiung « (Rath 2007: 66) erwirkte die Hansestadt Lübeck für die Küsten Dänemarks im Jahr 1204. Ohne solche Abkommen hätte der Städtebund überhaupt nicht funktionieren können. Nach diesem Abkommen gehörte den Schiffbrüchigen alles, was sie selber bergen konnten; der Rest fiel an die Strandbewohner. Um allerdings »trotzdem in den Besitz aller angetriebenen Güter zu kommen, brauchten die Strandbewohner nur abzuwarten und für die um ihr Leben kämpfenden Seeleute keine Hand zu rühren.« (Ebd.) Das ist sozusagen eine unmetaphorische Version des »Schiffbruch[s] mit Zuschauer « (Blumenberg 1979). Noch aus der zweiten Hälfte des 19. Jahrhunderts sind Fälle überliefert, in denen die Bewohner der Nordund Ostseeinseln Schiffbrüchigen aus ökonomischen Interessen heraus keine Hilfe gewährten. Als ein Schiff 1881 auf einer südschwedischen Insel auf Grund lief, reagierte niemand auf die Notsignale, erst als es einem Matrosen zwei Tage

1 | Der finnische Rechtshistoriker Vilho Niitemaa stellt fest: "Somit muss man das Strandrecht in seiner ursprünglichen Form, wonach ein Schiff, wenn es scheiterte und an den Stand trieb, mit seiner Besatzung, seiner Ladung und seinem sonstigen beweglichen Gut demjenigen zufiel, der von inm Besitz ergriff, als eine universale Auffassung ansehen, die die ältesten Rechtsanschauungen des Menschen widerspiegelt." (Niitemaa 1955: 18) 
später mit einem Floß gelang, das Land zu erreichen, »erschienen die Hilfsmannschaften am Ufer, die schon die ganze Zeit in den Dünen gelegen und das Geschehen auf dem gestrandeten Schiff beobachtet hatten.« (Rath 2007: 69)

In der Frühneuzeit haben die staatlichen Gewalten den Strand zunehmend $\mathrm{zu}$ hegen versucht und selbst ihren Anspruch auf diese Zone erhoben. Sie haben nach und nach Strandvögte eingesetzt, Strandordnungen erlassen und den sogenannten Bergelohn eingeführt. Im Allgemeinen Landrecht für die Preußischen Staaten von 1794 heißt es beispielsweise: »Hafen und Meeresufer, und was auf diese von der See angespült oder ausgeworfen wird, sind nach gemeinen Rechten ein Eigenthum des Staats.« (ALR 1794, Teil II, 15: \80) Es gibt »zur Beobachtung des Strandes angesetzte Beamt[e]«, die dafür zu sorgen haben, »daß gestrandete Sachen gerettet, erhalten, und den Eigenthümern zurückgegeben werden.« (Ebd.: \82) »Gestrandete Sachen« hingegen, »zu welchen kein Eigenthümer sich meldet«, gehören nicht dem Finder, sondern »dem Staate« (ebd.: \86). ${ }^{2}$ Was dem Finder zusteht, ist lediglich ein in den Strandordnungen der Provinzen festgelegter »Bergelohn « (ebd.: $₫ 85) .^{3}$

Der Strand 4 ist eine »elementare Übergangszone« (Wolf 2013: 297) zwischen Land und Meer. Er gehört noch nicht zum mare liberum (vgl. Grotius 1916), zum >ökonomisch-theologisch< konzipierten Meer, das alle Schiffe - als Vorbereiter und Vorboten der globalisierten Wirtschaft - frei befahren dürfen, das ius communicationis für sich in Anspruch nehmend (vgl. Thumfart 2009: 26); und er ist auch nicht der Ort des geregelten Austausches zwischen Land und Meer, 5 der Hafen, wo die Schiffe in See stechen und die Ladungen gelöscht werden. Er ist wild. Aus der Perspektive der Globalisierung wie des Staates sind Strandbewohner daher strukturell rückständig und latent barbarisch. In einer Publikation des

2 | Sogar das Strandrecht im alten Sinne selbst wird - wenn man so will - im Allgemeinen Landrecht zu einem staatlichen Instrument: "Gegen fremde Nationen, welche das Strandrecht noch ausüben, behält sich der Staat eben dieses Recht, zur Schadloshaltung seiner verunglückten Unterthanen, ausdrücklich vor." (Ebd.: § 87)

3 | Weil immer wieder große Werte zu bergen waren, konnte allerdings auch der Bergelohn als Einkommensquelle durchaus ins Gewicht fallen: "Auf der Insel Amrum übertraf er mehr als einmal das Jahreseinkommen der gesamten Inselbevölkerung." (Rath 2007: 85)

4 | Es versteht sich von selbst, dass juristisch gesehen von alters her unklar war, was eigentlich genau unter dem Begriff des Strandes zu verstehen sei - insbesondere, wie weit der Strand ins Meer reichte. Hier eine Feststellung dazu (aus einem Rechtsgutachten zu einer einschlägigen Problematik): "Das Gewässer, über das die Strandhoheit sich ausdehnte, gehörte eben zum Strande und fiel mit unter den Begriff des Strandes. Ob man diesen Meeresgürtel als Küstengewässer, Strandgewässer, Strand oder Strandmeer [...] bezeichnen will, ist einerlei." (Strecker 1925: 10)

5 | Das berühmte Büchlein Land und Meer von Carl Schmitt (Schmitt 1993) beschäftigt sich zwar mit dem Land und dem Meer (mit der Ordnung des Landes und des Meeres), nicht aber mit der Frage nach der unklaren Grenzzone zwischen beidem. Das kann kaum verwundern, weil der Dezisionismus hier ein Schlag ins Wasser ist. 
preußischen Staatsrates von 1801 kommt das sehr gut zum Ausdruck. Der König habe »mißfällig wahrgenommen, daß die Verordnungen, welche von Zeit zu Zeit wegen des bei Strandungen [...] zu beobachtenden Verfahrens ergangen sind, von den Strandbewohnern in Westpreußen häufig außer Acht gelassen« würden; nicht selten werde »die Mannschaft eines verunglückten Schiffes« einem »lieblosen Verhalten « ausgesetzt, und immer wieder komme es zu »Räubereien«, was zum Teil allerdings »in der Unbekanntschaft der Strandbewohner mit den vorhandenen gesetzlichen Vorschriften « zusammenhängen möge, die im Folgenden noch einmal zusammengefasst würden (Simon 1844: 377). Es folgt in 19 Paragraphen ein System von Strafen und Belohnungen, das die - stets als ein >unter einer Decke< steckendes Kollektiv betrachtete - Strandbewohnerschaft disziplinieren soll. ${ }^{6}$ So ist »jeder Strandbewohner schuldig«, im Falle einer Strandung nach Kräften bei der Rettung von Mannschaft und Ladung mitzuwirken und den Anweisungen des »Strandbedienten « Folge zu leisten; vor erfolgter Rettung dürfen die Strandbewohner nicht mit den Schiffern in Unterhandlung in Sachen Bergelohn treten; der Bergelohn, den die Schiffer den Strandbewohnern entrichten müssen, darf bis zu einem Drittel des Wertes der geborgenen Sachen gehen; erweist sich ordnungsgemäß abgeliefertes Strandgut als herrenlos, erhält der Finder die Hälfte; wer nachts ein Feuer am Strand entzündet, welches Schiffe irreführen mag, kann mit Festungshaft zwischen einem bis zu zehn Jahren bestraft werden; wer sich unbefugt gewaltsam Zutritt $\mathrm{zu}$ einem gestrandeten Schiff verschafft, »soll mit geschärfter Züchtigung und Einsperrung in eine Besserungsanstalt bis auf drei Jahre belegt werden.« (Simon 1844: 378)

Die Abschöpfung eines - wie man es nennen könnte - Friktionsgewinns durch den Staat beschränkt sich nicht nur auf Transportgut, das in den Reibungen zwischen Land und Meer zu Strandgut geworden ist und nun eingesammelt werden kann. Das Strandgut steht hier im Regelfall in Verbindung mit einem konkreten Ereignis, das >im Idealfall< ein Wrack erzeugt,7 und die Dauer, während derer das zum Strandgut Gewordene tatsächlich dem Speichermedium Meer überlassen war, bleibt sehr begrenzt und kann völlig entfallen (wenn es das Wrack selbst ist, das ausgeraubt wurde). Wenn diese Dauer recht lang ist wenn also der Bezug zu einem konkreten Ereignis verblasst ist oder wenn es ein solches Ereignis gar nicht gegeben hat -, spricht man terminologisch eher von Treibgut, das ebensowohl Rückstände wie Wertgegenstände, ebenso Abfall wie

6 | "Der Strandraub ist Kollektivritus, schließlich betätigt sich die Gemeinschaft aus Anlass einer gemeinsamen Beute, dass sie mehr als ein Agglomerat von Individuen und atomisierten Interessen ist." (Wolf 2013: 299) Es geht hier eben nicht um das Verhältnis eines Einzelnen (eines Vereinzelten) am Strand, der ein ästhetisches (kontemplatives) Verhältnis zum Meer hat, sondern um ein Tun, bei dem auch der Einzelne als Teil eines Kollektivs wahrgenommen wird.

7 | Das Schiffswrack ist eine faszinierende Figur, die im Zentrum zahlloser literarischer Texte steht, wie man Burkhardt Wolfs eindrucksvoller Studie Fortuna di mare. Literatur und Seefahrt entnehmen kann (vgl. vor allem Wolf 2013: 294-296). 
Naturgegenstände umfassen kann. Die obrigkeitliche Abschöpfung hat sich aber nicht nur auf Wirtschaftsgüter bezogen, sondern auch auf Dinge, die >das Meer selbst < hergibt, die also nicht >geschöpft< wurden - Dinge also, die keine Sachen waren, die nie einen >Herrn $<$ hatten. Sie müssen in einem anderen Sinne und auf eine andere Art gesammelt werden. ${ }^{8}$

Dass es solche Dinge überhaupt gibt, zeugt von dem besonderen Gewaltverhältnis, ${ }^{9}$ das die Beziehungen zwischen den Strandbewohnern und den obrigkeitlichen Institutionen grundiert. In der preußischen Publikation für die Strandbewohner von 1801 schließt sich an den ersten, dem eigentlichen Strandgut gewidmeten Abschnitt ein zweiter Abschnitt an, welcher »der Befugniß, Bernstein zu suchen, und den bei dessen Auffindung zu beobachtenden Pflichten « (Simon 1844: 378) gewidmet ist. Bernstein kann in Europa als das Paradigma eines wertvollen Naturprodukts gelten, das, obwohl vom Meer abseits der staatlichen Territorien und jenseits aller Eigentumsverhältnisse >hergegeben $<$ gleichwohl nicht vom Finder angeeignet werden darf. Bernstein ist ein fossiles Harz. Die insbesondere in der Ostseeregion vorkommenden Bernsteinarten haben ein Alter von 30 bis 50 Millionen Jahren; das Meer hat den Bernstein also sehr lange gespeichert, bevor er - vor allem nach Stürmen - in den Uferbereich gespült und schon in vorgeschichtlicher Zeit als Schmuckstein gesucht und gefunden wurde (gesammelt, aber nicht eingesammelt). Gleich der erste Paragraph der Publikation stellt fest: »Der Bernstein ist ein vorbehaltenes Eigenthum des Staats.« (Ebd.: 379) Wer unbefugt Bernstein findet, muss ihn abgeben und bekommt den zehnten Teil seines Werts, wer ihn unbefugt sucht, wird als Dieb bestraft. Wer einen Bernsteindieb erfolgreich denunziert, bekommt ein Viertel des Wertes.

Es ist nicht verwunderlich, dass die Strandbewohner so etwas nicht gerne lasen. Dass sie aber vom Eigentumsanspruch des Fiskus auf den Bernstein nichts wüssten, kann man nicht behaupten. Tatsächlich datieren die Bernsteinregalien für den Ostseebereich zumindest aus dem Hochmittelalter, genauer gesagt, sind sie verbunden mit der von den Kreuzzügen zurückgekehrten Ritterschaft des Deutschen Ordens, die nach ihrer Eroberung des Samlandes das einträgliche Recht auf das Bernsteinsammeln für sich in Anspruch nahm. Im weiteren Verlauf wurden die »Strandbewohner [...] verpflichtet, den Bernstein zu sammeln, zu schöpfen, zu stechen und den Ordensbeamten abzuliefern, welche ihnen ihre geringe Bezahlung aushändigten.« (Tesdorpf 1887: 9) Wilhelm Tesdorpf, der im 19. Jahrhundert eine historische Studie zum Bernsteingewinn in

8 | Vgl. für eine elaborierte Theorie Manfred Sommers Sammeln. Ein philosophischer Versuch (2002).

9| Der juristische Begriff des besonderen Gewaltverhältnisses betrifft Verhältnisse zwischen Subjekt und Staat, in denen ein besonderer Status vorliegt, in welchem die bürgerlichen Grundrechte teilweise außer Kraft gesetzt sind (das ist sowohl bei Beamten wie bei Strafgefangenen oder Soldaten der Fall). Kennzeichen eines besonderen Gewaltverhältnisses sind insbesondere Disziplinarmaßnahmen (vgl. Niehaus 2003: 358-360). 
Preußen verfasst hat, wirft natürlich die Frage auf, wie sich die Ordensritter gegen Unterschlagungen schützen konnten. Er sieht, mit eindeutiger Sympathie für die Strandbewohner, einen über Jahrhunderte währenden Wettbewerb zwischen Vorbeugungsmitteln und Unterschleif: Auch wenn auf den »Bernsteindiebstahl die härtesten Strafen gesetzt waren, obwohl der Ergreifung bei unbefugtem Bernsteinsammeln Aufknüpfen am nächsten Baum auf dem Fuße zu folgen pflegte« (ebd.), hätten die Samländer nicht aufgehört, Bernstein beiseitezuschaffen. »Die Korrumpierung, die so notwendig entstand, stieg je länger, desto mehr«, wovon die »von Jahrhundert zu Jahrhundert verschärften Strandordnungen und Maßregeln« (ebd.) Zeugnis ablegten. Diese Argumentation setzt voraus, dass die Strandbewohner zu Recht kein Unrechtsbewusstsein hatten, wenn sie Bernstein suchten und fanden. Dass man in einem extraterritorialen Raum, in dem alle Eigentumsverhältnisse suspendiert sind, sammeln darf, scheint zu den unverbrüchlichen >natürlichen< Rechten zu gehören, zu einer $>$ natürlichen Einstellung<, die nicht ausgerottet werden kann. ${ }^{10}$

In Zeiten der Absatzkrise - zum Beispiel als nach der Reformation nicht mehr so viele Rosenkränze aus Bernstein benötigt wurden - wurden die Gewinnungskosten überdies dadurch gedrückt, dass die Strandbewohner »statt mit Geld mit Salz« (Tesdorpf 1887: 12) bezahlt wurden (auf welches die Regierung ein Monopol hatte). Überdies wurde ein Verwaltungsapparat um den Bernsteingewinn aufgebaut, der im Lauf der Zeit weiter verfeinert wurde, »um eine desto genauere Überwachung des ganzen Strandes zu ermöglichen.« (Ebd.: 17) Ende des 16. Jahrhunderts hatte man außerdem »längs des Strandes Galgen« (ebd.: 20) für die sofortige Exekutierung unbefugter Bernsteinsammler errichtet. Das besondere Gewaltverhältnis schlug sich aber auch darin nieder, dass man allen erwachsenen Strandbewohnern - in Preußen etwa nach der Bernsteinordnung

10 | Darin liegt mehr als die Feststellung eines Gewohnheitsrechts. Gegen eine ,bloß, gewohnheitsrechtliche Anschauung (dass man beispielsweise Strandgut einsammeln darf) kann man Argumente geltend machen (etwa dass der noch lebende Eigentümer seine Rechte nicht aufgegeben hat), gegen die Anschauung, dass man sammeln darf, was der extraterritoriale Raum (die 'Wildnisı) selbst hergibt, kann man nur mit Gewalt einschreiten. Das sogenannte Unrechtsbewusstsein kann in diesem Fall keine Wurzeln schlagen. In abgeschwächter Form zeigt sich dies auch im Verhältnis der reinfachen Bevölkerung، zum Wald, in dem man Reisig usw. sammeln darf. Das Parallelbeispiel des Waldes zeigt aber auch: Man muss erst einmal etwas als extraterritorial definieren, um diese ,natürliche Einstellung، geltend machen zu können. Immanuel Kant - der bekanntlich in Königsberg an der Ostsee lebte - erwähnt die rechtliche Frage des Bernsteinsammelns im Zusammenhang mit der Frage, wie weit das eigene Territorium in Bezug auf das nichtterritoriale Gewässer reiche. Seine Antwort ist pragmatisch: "So weit ich aus meinem Sitze mechanisches Vermögen habe, meinen Boden gegen den Eingriff anderer zu sichern (z.B. so weit die Kanonen vom Ufer abreichen), gehört [der Boden] zu meinem Besitz und das Meer ist bis dahin geschlossen (mare clausum)." (Kant 1993: 381 [A 95]; Hervorh. i.0.) Das Kollektiv der Küstenbewohner kommt in dieser Erwägung freilich gar nicht vor. 
des Großen Kurfürsten im 17. Jahrhundert - alle paar Jahre sogenannte Strandeide abverlangte: Sie mussten schwören, keinen Bernstein zu entwenden und alle ihnen bekannt werdenden Unterschlagungen unverzüglich anzuzeigen (vgl. ebd.). Parallel dazu war Ortsfremden, die den Eid nicht geleistet hatten, überhaupt der Zugang zum Strand untersagt - ein Verbot, das in Ostpreußen erst 1885 aufgehoben wurde.

Kein Tourist am Strand, keine Strandspaziergänge: Auch wenn wir alle inzwischen - ob Touristen oder nicht - Bernstein suchen und finden können, wie es uns beliebt, sollte aus dieser Vorgeschichte deutlich geworden sein, dass der Strand nicht nur deshalb nicht als ein idyllischer Ort gelten kann, weil im Ozean die Ungeheuer hausen. Wie es vormals >wirklich< an den Stränden ausgesehen hat, ist damit nicht gesagt. Entscheidend ist vielmehr, dass der Strand nur in dieser Weise vom Diskurs erfasst werden konnte. Konkret kommt der Strand in rechtlichen Verordnungen, in Überlieferungen - nur als ein Ort der Friktion, der Friedlosigkeit, des Kampfes, als zweideutige Zone vor. ${ }^{11}$

\section{Strandspaziergang}

Wer sich als ein Einzelner an den Strand begibt, ohne zum Kollektiv der Küstenbewohner zu gehören, hat zunächst und zumeist kein konkretes ökonomisches Interesse. Seine Einstellung kann, insoweit es ihm um den Strand als solchen geht, im weiteren Sinne als ästhetisch bezeichnet werden. Zum Ort solch >interesselosen Wohlgefallens < im Sinne Kants wird der Strand insbesondere beim Strandspaziergang. Wer einen Strandspaziergang macht, verspürt nichts von der friedlosen Vergangenheit des Strandes, sie ist ihm allenfalls als ferne Erinnerung präsent.

Das siebte Kapitel von Thomas Manns Zauberberg ist mit »Strandspaziergang« überschrieben. Berühmt ist dieser Abschnitt, weil sich Thomas Mann in einem abermaligen essayistischen Exkurs die Frage vorlegt, ob und inwiefern man »die Zeit erzählen « (Mann 1981: 654) kann. In diesem Rahmen ist ihm der Strandspaziergang jene besondere, durch »landschaftliche Umstände « gegebene »Lebenslage«, in der sich das Subjekt einer »Verwirrung und Verwischung der zeitlich-räumlichen Distanzen bis zur schwindligen Einerleiheit« (ebd.) aussetzen kann. Ein derartig verzaubernder >anderer Zustand k könne freilich »für Ferienstunden allenfalls als statthaft gelten« (ebd.). Wenn der Erzähler (im Plural von sich sprechend) erklärt, er vertraue darauf, »daß auch Erfahrung und Erinnerung des Lesers uns nicht im Stich lassen werden, wenn wir auf diese wunderbare Verlorenheit Bezug nehmen« (ebd.: 661), appelliert er an ein Kollektiv anderer Ordnung - an ein Kollektiv der Einzelnen, die in Ferienstunden schon

11 | Zu diesem Komplex gehören natürlich neben dem unmittelbaren Strandraub noch weitere - hier nicht in den Blick genommene - Delikte und Maßnahmen, die für die Grenze zwischen Land und Meer charakteristisch sind, so etwa der Schmuggel auf der einen oder der Bau von Leuchttürmen auf der anderen Seite. 
einmal als Vereinzelte die unpezifische (aber unschwer als nichtmediterran erkennbare) Meeresentgrenzung erfahren haben:

Sausende Öde, blaß hellgrau überspannt, voll herber Feuchte, von der ein Salzgeschmack auf unseren Lippen haftet. Wir gehen, gehen auf leicht federndem, mit Tang und kleinen Muscheln bestreutem Grunde, die Ohren eingehüllt vom Wind, von diesem großen, weiten und milden Winde, der frei und ungehemmt und ohne Tücke den Raum durchfährt und eine sanfte Betäubung in unserem Kopfe erzeugt, - wir wandern, wandern und sehen die Schaumzungen der vorgetriebenen und wieder rückwärts wallenden See nach unseren Füßen lecken. (Ebd.)

Ersichtlich wird hier versucht, den Strandspaziergang als eine ästhetische Situation zu beschwören, die zwar alle Sinne erfasst, aber keinen überwältigt und die uns daher auf eine integrale Weise absorbiert. Das ist die Voraussetzung der »Einerleiheit«. Das »sanft brausende Getöse« der Brandung »sperrt unser Gehör für jede Stimme der Welt«, und daraus resultiert »[t]iefes Genügen, wissentlich Vergessen« (ebd.). Wir sollen uns also daran erinnern, wie wir uns am Strand dem Vergessen anheimgeben. Der Text versetzt uns in die Situation am Strand: »Schließen wir doch die Augen, geborgen von Ewigkeit! Nein, sieh, dort in der schaumig grau-grünen Weite, die sich in ungeheuren Verkürzungen zum Horizont verliert, dort steht ein Segel. Dort? Was ist das für ein Dort? Wie weit? Wie nah?« (Ebd.) Wir können die Entfernung des entfernten Segelbootes nicht schätzen, weil wir »kein Organ und Sinn« haben, das uns »über den Raum Bescheid « (ebd.) gibt. Und diese Dislozierung spiegelt sich in unserer eigenen Dislozierung, die Raum und Zeit verschwimmen lässt: „Wir gehen, wir gehen, wie lange schon? Wie weit? Das steht dahin. Nichts ändert sich bei unserem Schritt, dort ist wie hier, vorhin wie jetzt und dann; in ungemessener Monotonie ertrinkt die Zeit« (ebd.).

Ein solcher Strandspaziergang hat zunächst einmal keine Affinität zum Strandgut. Weder richtet das postulierte Subjekt seinen schweifenden Blick in die Ferne, um gegebenenfalls der Anzeichen eines schiffbruchartigen Ereignisses gewahr zu werden, noch heftet es den schweifenden Blick bisweilen vor sich auf den Boden, um vielleicht etwas Kleines zu entdecken und sich gegebenenfalls zu bücken. Nur aber, wer in gleichsam freischwebender Aufmerksamkeit Letzteres tut (wer sich nicht vollständig in die »Einerleiheit« fallen lässt oder sich ihr nicht ganz überlassen kann), wendet sich, ohne dabei unbedingt etwas Bestimmtes zu suchen, darüber hinaus dem sinnlich Konkreten zu. Dann öffnet sich das Subjekt dem Spiel der Einbildungskraft, und das Meer wird als Speichermedium auch zu einem Speichergedächtnis, das unablässig etwas auf dem Strand ablagert, was möglicherweise (jenseits ökonomischer Interessen) aufgelesen werden kann, was vielleicht zu Assoziationen anregt oder Erinnerungen weckt.

Dass derjenige, der am Strand entlanggeht, bereit sei für einen Fund, ist in der Ära des befriedeten Strandes untrennbar mit der >Institution< des als ein »Umherstreifen am Meeresufer« (Corbin 1990: 225; Hervorh. i.O.) verstandenen 
Strandspaziergangs verknüpft. Die Zone des einsamen Strandes ist in unserer Kultur der exemplarische Ort geworden, an dem man etwas finden kann, ohne etwas Bestimmtes gesucht zu haben. Die Subjektposition, die im Umherstreifen am Strand impliziert ist, muss in Verbindung zur Kulturtechnik des Spazierengehens gesehen werden, das um 1800 als eine Gelegenheit zum »freye[n] Spiel der Vorstellungskräfte« (Schelle 1990: 50) hypostasiert wird: »Der Mensch ist nur da ganz Mensch, wo er spazierengeht«, fasst Harald Neumeyer (1999: 9) zusammen. An Spaziergänge am Strand ist damit allerdings zunächst noch nicht gedacht (vgl. König 1996; Moser/Schneider 2007). ${ }^{12}$ Denn der Spaziergang zu Lande als ein müßiges Tun ist zwar nicht wie die Promenade eine Veranstaltung, um zu sehen und gesehen zu werden, findet aber gleichwohl in einem von der Kultur kodierten Raum statt. Die Freiheit des kultivierten abendländischen Spaziergängers, sich ablenken zu lassen, seine Offenheit gegenüber unvorhergesehenen Eindrücken, nimmt im Strandspaziergang eine ganz andere Form an. Erstens ist dem Spaziergänger am Strand die Richtung vorgegeben, zweitens ist seine Offenheit für Eindrücke von einer Monotonie grundiert, die zu einer Zerstreuung dieser Eindrücke führt, drittens wird der Strandspaziergang natürlich in anderer Weise als ein Randgang zwischen Natur und Kultur empfunden, und viertens ist er mit der Bereitschaft des Auflesens von Dingen verknüpft. In einigen dieser Aspekte erweist sich der Strandspaziergänger als Spiegelbild des seit langem als paradigmatisches modernes Subjekt aufgefassten Flaneurs, obwohl (bzw. weil) dieser umgekehrt ganz und gar im Zivilisationsraum angesiedelt ist (vgl. Neumeyer 1999). In Ansehung dessen darf man überspitzt formulieren: Der Mensch ist dort, wo er am Strand spazieren geht, ganz und gar ein Geschöpf der modernen westlichen Zivilisation. Und deshalb kommen in Ansehung unseres Verhältnisses zu den Dingen, die da zu finden sind, ganz basale Kategorien zum Einsatz.

Zunächst einmal hat der Umstand, dass das, was das Meer dem Strandspaziergänger vor die Füße gespült hat, nicht als ein Wirtschaftsgut angesehen wird (dass es Treibgut ist), verschiedene Implikationen. Das Meer hat dafür gesorgt, dass es für uns keine Sache ist, sondern ein Ding. Und dieses (nicht zuletzt vom Meer gesäuberte) Ding siedelt sich, sobald es von uns wahrgenommen wird, grob gesagt, zwischen den Polen Abfall und Souvenir an. Als Abfall gilt, was sich nicht mehr verwerten lässt. Als Souvenir gilt, was einen Erinnerungswert bekommen kann. Am Strand werden in erster Linie Rückstände der modernen Zivilisation - vor allem >hässlicher < Plastikmüll - als Abfall aufgefasst. Zu möglichen Souvenirs hingegen werden in erster Linie >schöne Naturdinge wie Muscheln usw. gezählt. Insofern kommen also ästhetische Kategorien verbunden mit einer moralischen Wertung zur Anwendung. Der konkrete Zeichen- oder Verweischarakter des Strandguts bzw. Treibguts realisiert sich allerdings erst jen-

12| Heinrich Heine beispielsweise, der in den Reisebildern auch von seinem Besuch auf der Insel Norderney berichtet, erklärt zwar, dass er gerne am Strand spazieren geht, gedenkt dort aber nur der "seemännischen Wundersagen" (Heine 1826: 137); von Strandgut oder Treibgut ist keine Rede. 
seits dieses Gegensatzes zwischen dem Abfall als »undifferenzierte[r] Materialmasse (Weber 2014: 157) und Einzelstück als »mobile[m] Gedächtnismedium « (Holm 2014: 197). Das einzelne (reingewaschene) Müll-Ding verweist auf etwas Vergangenes und Entferntes, während umgekehrt das Souvenir erst in der Zukunft und in einiger Entfernung auf die Zeit am Strand verweisen wird. Weil aber das Meer (sofern es kein Binnenmeer ist) an alle Länder grenzt, vermag es die Einbildungskraft von uns Angehörigen der westlichen Zivilisation zu entgrenzen. In dem, was es als Strandgut bzw. Treibgut an den Strand wirft, bringt das Meer sich selbst als Speichergedächtnis in Erinnerung, aber in mehrfacher Vermittlung auch das, woran es grenzt.

\section{Meer als globaler Erinnerungsraum}

Zu Beginn seines Büchleins Kleines Strandgut, das er zusammen mit seiner Frau als Illustratorin verfasst hat, verleiht Siegfried Lenz seinem immer wiederkehrenden Wunsch Ausdruck, dass das Meer »plötzlich, in einer Nacht - schrumpfte und austrocknete und seinen runzligen Grund freigäbe mit allen Geheimnissen « (Lenz/Lenz 2011: o.S. [3]), dass wir also das Innere dieses Speichermediums selbst mit unseren Blicken erfassen könnten. Da die Erfüllung dieses Wunsches sogleich als unmöglich erkannt wird, muss sich das Ich damit begnügen, als »Sucher und Sammler « die »Geschenke« in Augenschein zu nehmen, »die das Meer dem Strand macht.« (Ebd.: o.S. [5]) Diese Dinge, die uns das metaphorisch als Interaktionspartner aufgefasste Meer »vor die Füße spült«, erschienen nur »auf den ersten Blick [...] ziemlich wertlos«, nämlich als »ausgespiene Nutzlosigkeiten, Reste, zernagte Belanglosigkeiten, die dem Meer nichts bedeuten.« (Ebd.) Eine solche Geringschätzung habe »das Strandgut nicht verdient«; vielmehr sei »jedem Stück etwas abzuhorchen, abzusehen, auch das unscheinbarste, alltäglichste ist bereit, etwas zu erzählen« (ebd.) - so die Behauptung, welche die Auswahl kleiner Betrachtungen in diesem schmalen Band unter Beweis stellen soll.

Ein »Tauende«, eine »Buddel«, ein »Knochen« oder ein »pockenbesetzter Pott« (ebd.: o.S. [7]), aufgelesen und »träumerisch gegen den Horizont gehalten«, laden uns ein, »ihrer Herkunft nachzudenken« (ebd.), sie appellieren »an unsere Phantasie, mögliche Schicksale zu entwerfen«, »weil alles, was wir am Saum des Meeres finden, etwas bezeugt: einen Unfall, ein Versehen, Leichtsinn und Übermut, Gewalt und ordnungsgemäßen Tod.« (Ebd.: o.S. [7f.]) Der Strand wird also in einen Ort poetischer Imagination verwandelt, die sich an den Dingen konkretisiert und bewährt, die am Strand zu finden sind: »Was du hier am Strand erfahren willst, mußt du dir erfinden, denn du magst noch so viel entdecken und sammeln: über seine Herkunft schweigt jedes Ding.« (Ebd.: o.S. [35]) Dabei versucht Siegfried Lenz, auch die Unterscheidung zwischen Naturgegenständen und Zivilisationsrückständen zu verflüssigen. Denn die einen wie die anderen hat das Speichermedium Meer nach und nach modifiziert. Während die gestrandete Fracht aus ökonomischen Gründen der materiellen Werterhaltung 
möglichst unverdorben (und zügig) geborgen werden musste, gewinnt das >kleine Strandgut< - das Strandgut als Treibgut - genau umgekehrt dadurch an immateriellem Zeichen-Wert, dass es Spuren der allmählichen Bearbeitung durch das Meer zeigt: »Wer den Ast brach, den die Wellen ans Land werfen, kann keiner ermitteln, doch du fühlst dich aufgefordert, dem weißen, gepökelten Holz eine Reise zu entwerfen, zu denken, welche Strömungen es entführten « (ebd.). Während die Begegnung von Meer und Strand einige Relikte - wie die »Reste der Krabben« (ebd.: o.S. [23]) - nach und nach zerreiben wird, hat das Meer die Seeigel, damit sie »für immer Seeigel bleiben«, konserviert, indem es sie »zu Stein« (ebd.: o.S. [39]) gemacht hat.

Nicht nur die Naturgegenstände, die das Meer an den Strand spült, werden von ihm diesen gleichsam naturgeschichtlichen Prozessen der Modifikation unterworfen, sondern auch die Artefakte der Zivilisation. Unter anderem werden ein Schuh, eine Schrubbbürste, eine zerbeulte Dose, eine Injektionsspritze, ein Plastikhelm, ein Stück Ankertau und leere Schrotpatronen für Lenz zum Ausgangspunkt von kurzen Betrachtungen oder kleinen Geschichten. Dabei deutet sich freilich an, dass der Strandspaziergang selbst zwar ein Ort der Kontemplation sein mag, dass aber der Strand deswegen noch nicht zu einem Ort der Harmonie geworden ist. Eine von einem Ausflugsdampfer ins Meer geworfene »Limonadendose«, so Lenz, habe »im Meer ebensowenig zu suchen wie eiserne Rohrstücke oder eine Stabtaschenlampe« (ebd.: o.S. [57f.]), und umgekehrt wirft das Meer auch den toten Alk mit »verklebtem Gefieder « und »Öl in seinen Lungen « (ebd.: o.S. [83]) an Land oder die »von den Schwermetall-Wunden bedeckten Fische«, die beim Fang »bereits auf See ausgesondert wurden« (ebd.: o.S. [87]).

Wenn das Meer in dieser Weise an die Kollateralschäden der Zivilisation erinnert, dann geschieht dies unter Absehung aller interkulturellen Unterschiede. Oder anders ausgedrückt: Der Abfall der westlichen Zivilisation ist global. Auf den Meeren gibt es keinen anderen Abfall - auch wenn jede »materielle Kultur [...] sowohl durch den Umgang mit den Dingen als auch durch ihr Entsorgen geprägt ist.« (Weber 2014: 157) Aus der Perspektive des Strandes und der hard facts ist das Meer kein interkultureller oder transkultureller, sondern ein globaler Erinnerungsraum. In dem kleinen Buch von Lieselotte und Siegfried Lenz wird dies noch auf recht selektive Weise vorgeführt, insofern es - jeweils auf der linken Doppelseite gezeichnete - Einzelstücke sind, die einen Resonanzraum eröffnen. Die Massenhaftigkeit des Zivilisationsabfalls bleibt bei diesem poetischen Strandspaziergang ausgespart, und ebenso spielt der prosaische Plastikmüll, dessen Widerstandskraft gegen die Erosion des Meeres seit Jahrzehnten im Fokus steht, nur eine untergeordnete Rolle.

Ihm widmen sich andere, gänzlich unpoetische Arbeiten wie etwa das Buch Strandgut aus Plastik und anderer Meeresmüll (Timrott 2015). Der Meeresmüll besteht nach offiziellen Schätzungen zu etwa 70 Prozent aus Kunststoffen (vgl. ebd.: 14), die das Meer erst nach Jahrzehnten, teilweise auch erst nach Jahrhunderten in (nicht weniger schädliche) Mikropartikel zersetzt und zuvor auf der ganzen Welt verteilt hat. Porträtiert wird dann - ausgehend von der Situation in 
der Nordsee - Gewerbemüll (Netzfragmente, Arbeitshandschuhe, Gehörschutzstöpsel, Plastikfolien usw.), Müll aus Freizeitaktivitäten (Luftballons, Spielzeug, Feuerwerkskörper, Angelzubehör usw.) und Verbrauchermüll (Getränkeflaschen, Joghurtbecher, Kosmetikbehälter, Hundekotbeutel usw.). ${ }^{13}$ Im kulturübergreifenden Plastikmüll an den Stränden bringt sich die Globalisierung in Erinnerung. Die lokalen Strände, an denen das Strandgut vornehmlich aus Naturgegenständen besteht, sind die >künstlichen Paradiese «, an denen der Abfall (in der Saison) ohne jegliches >interesseloses Wohlgefallen < systematisch - also von Nichtstrandspaziergängern - eingesammelt wird. Insofern sind die Strände >von Natur aus< global und vermüllt.

Die westliche Zivilisation ist freilich nicht nur diejenige, die in der Globalität des Strandguts jenseits aller interkulturellen Differenzen ihre Vorherrschaft bekundet, sie ist auch diejenige, die den Raritäten des Strandguts Museen errichtet und das Strandgut zum gesellschaftlichen und wissenschaftlichen Thema gemacht hat. ${ }^{14}$ Und sie hat es in gewisser Weise sogar als ein Mittel der Forschung eingesetzt, insofern der Umstand, dass Strandspaziergänger offen für das Auflesen und Inspizieren von Strandgut sind, dazu genutzt wurde, die Eigenschaften von Meeresströmungen selber genauer zu untersuchen. Bereits zu Beginn des 19. Jahrhunderts kam man auf den Gedanken, die in der Folklore des Meeres einen so vornehmen Platz einnehmende Institution der Flaschenpost für diese Zwecke zu verwenden. Die Flaschenpost ist sozusagen der singuläre Fall eines Treibguts, das zur konkreten Botschaft wird, in der sich jemand in Erinnerung bringt - und insofern der Modellfall eines Briefes, der immer seinen Bestimmungsort erreicht (vgl. Lacan 1975), weil jeder, der ihn lesen kann, ein korrekter Adressat ist. Um beispielsweise die Geschwindigkeit des Golfstroms genauer zu untersuchen, versenkte man im 19. Jahrhundert größere Mengen von Flaschenposten im Meer, in denen nebst genauer Datierung und Lokalisierung des Absenders im Falle des Auffindens in verschiedenen Sprachen um Rückmeldung gebeten wurde. Allerdings kann der rechte Zeitpunkt vorübergegangen sein, sodass die Rückmeldung nur noch den Wert eines anekdotischen Belegs dafür hat, dass das Unwahrscheinliche geschieht und das Medium die Botschaft ist. Einen wissenschaftlichen Hintergrund hatten auch die Flaschenposten, die 2015 auf

13 | Der ausführliche Wikipedia-Artikel Plastikmüll in den Ozeanen gibt über die vielfältigen Aspekte dieses Problems Auskunft. Nach offiziellen Schätzungen gelangen jährlich ca. 5 bis 12 Millionen Tonnen Plastikrückstände in die Meere. Nur etwa 15 Prozent kehren - wenn man so sagen darf - zurück an die Küsten. Die globale Dimension des Plastikmülls zeigt sich auch darin, dass sich in dem berüchtigten Müllstrudel Great Pacific Garbage Patch mehr als 100 Millionen Tonnen Kunststoffmüll befinden sollen und dass der Großteil des gesamten Plastikmülls durch nur zehn Flüsse in die Meere eingeleitet wird, von denen sich acht in Asien und zwei in Afrika befinden. Vgl. Wikipedia 2020c.

14 | Auf den niederländischen Nordseeinseln gibt es teilweise kleine Museen, in denen Kuriositäten unter den im Laufe der Jahre am Strand gefundenen Dingen präsentiert werden, so etwa im Juttersmuseum Flora auf Texel (vgl. VVV Texel o.D.). 
der Nordseeinsel Amrum und 2018 auf einer australischen Insel von Strandspaziergängern gefunden wurden. Erstere war als eine von tausend Flaschen 1906 im britischen Plymouth über Bord geworfen worden, Letztere 1886 von einem deutschen Frachtschiff (vgl. Wikipedia 2020a).

Die Multiplikation der ausgegebenen Posten führt zugleich vor Augen, dass das Meer ein Speichermedium ist, welches die Dinge nicht nur zeitversetzt, sondern auch in unwahrscheinlicher Streuung zustellt. Das kann sich nämlich nur zeigen, wenn der >Ausgabeort< bekannt ist. Das berühmteste Beispiel sind die sogenannten friendly floatees. Am 10. Januar 1992 verlor ein Frachtschiff auf der Fahrt von Hongkong an die amerikanische Westküste nahe der Datumsgrenze im Pazifik bei einem Sturm drei Container, wobei knapp 30.000 Kunststofftiere der Firma First Year Inc. (gelbe Enten, grüne Frösche, blaue Schildkröten und rote Biber) ins Meer gespült wurden. In den folgenden 15 Jahren wurden nicht nur Exemplare in Australien und Indonesien sowie an der amerikanischen Westküste und in Chile, sondern auch - erstaunlicher Weise offenbar die legendäre Nordwestpassage durch das Packeis hinter sich - an der amerikanischen Ostküste sowie schließlich im Jahr 2007 sogar an der südenglischen Küste gefunden, ca. 27.000 Kilometer vom >Ausgabeort< entfernt (vgl. Wikipedia 2020b). All dies wird nur erfasst, weil es Strandspaziergänger auf aller Welt gibt, die etwas auflesen.

Ins Reich der schönen Literatur gehört es hingegen, wenn ein müßiger (aufnahmebereiter) Spaziergänger etwas am Strand findet, das ihn selber betrifft. An die Stelle eines pauschalen Adressiertseins durch die Flaschenpost tritt dann ein persönliches Adressiertsein, das allerdings strukturell gesehen auch nur vom Adressaten als solches erkannt werden kann und das Gegenstück zur globalen Adressierung ist. ${ }^{15}$ Was das Meer in diesem Falle vor die Füße spült, kann nicht nicht als Botschaft aufgefasst werden. Freilich kann diese Botschaft nicht beantwortet werden. Das Meer ist kein Gegenüber, mit dem es einen geregelten Austausch gibt.

In dem schmalen Buch Aus dem Leben einer Matratze bester Machart des Schweizer Autors Tim Krohn werden, wie es der Titel verspricht, beginnend mit dem Jahr 1935 und endend mit dem Jahr 1992, acht Stationen aus der Objektbiographie einer Matratze erzählt. Die erste und die letzte Station bilden eine Klammer: In der ersten Station wird erzählt, wie der deutsche Jude Immanuel Wassermann in der Schweiz eine junge Sizilianerin namens Gioia kennenlernt und vom Fleck weg heiratet; in der Hochzeitsnacht im Hotel gerät ein Blutfleck auf die nagelneue Matratze, der seltsamerweise die Form des amerikanischen Kontinents aufweist und ob dieses peinlichen Makels vom Ehemann kurzerhand käuflich erworben wird. In der letzten Episode begegnen wir Immanuel Wassermann noch einmal, 57 Jahre später. Er ist jetzt Pensionär in dem kleinen Ort

15 In der reinen Form ، dieser Struktur ist das adressierte Subjekt auch selbst der 'Ausgabeort: Das dem Meer überantwortete Ding wird - zum Subjekt zurückkehrend zur Botschaft, so idealtypisch in Schillers Ballade Der Ring des Polykrates (vgl. Niehaus 2009: 53-55), freilich unter Umgehung des Strandspaziergangs. 
Beaulieu unweit von Nizza und macht »täglich mehrmals seine >Strandrunde wie er das nannte« (Krohn 2014: 111):

Den Winter über wurde der Strand kaum gepflegt, das Treibgut häufte sich: allerlei weggeworfenes Material, zerstört oder sonstwie unbrauchbar geworden. Doch in Immanuel Wassermanns Augen wirkten die Dinge in ihrer Zersetzung und Verformung weit schöner, liebenswerter, gar lebendiger als in ihrer ursprünglichen, monotonen, zweckgerichteten Makellosigkeit. Im Treibgut erkannte er sich selbst, auch er war Abfall, zersetzt und verformt, seines ursprünglichen Zwecks und Nutzens beraubt, in gewissem Sinne längst schon tot. (Ebd.: 111f.)

Wassermann hat es sich angewöhnt, das Treibgut, angesichts dessen er manchmal »vor Rührung weinte« (ebd.: 112), aufzusammeln, es im Garten seiner Pension zu verbrennen und »aus den unbrennbaren Resten sonderbare Skulpturen $\mathrm{zu}$ formen, die er endlich, wenn er wieder Platz brauchte, von der Mole aus im Meer versenkte« (ebd.: 118). An diesem Morgen findet er die Reste der Matratze am Strand. Und er erkennt den immer noch sichtbaren Blutfleck: »den Golf von Mexiko, Zentralamerika, die Staaten der Karibik« (ebd.: 114). An diese Gestade jenseits des Meeres ist Wassermann niemals gelangt, wohl hingegen Gioia, deren Ehe mit ihm nach wenigen Tagen aufgrund der Nürnberger Rassengesetze annulliert wurde. Wassermann, der Überlebende der Konzentrationslager, blickt auf die Matratze am Strand - diese stumme Aufforderung zum Ziehen einer »Lebensbilanz« (Corbin 1990: 214) - und versucht, etwas zu empfinden, »angesichts des sonderbaren Zufalls«, dass er an diesem gleichgültigen Ort und

in einer Zeit, die nichts mehr mit jener zu tun hatte, in der sie sich getroffen und ihre Hochzeitsnacht begangen hatten, das Blut seiner Frau sah, Blut, das sie in einem Akt vergossen hatten, den er nicht einmal mehr bruchstückhaft erinnerte. Die Jahre der Gefangenschaft danach, der Folter, des Tötens (denn auch er hatte getötet) hatten alles andere verdrängt. (Krohn 2014: 115)

Schließlich verbrennt Wassermann, der seiner eigenen Lebensbilanz zufolge zum Treibgut Gewordene, die Matratze, das Treibgut, am Strand; und damit erinnert er uns daran, dass Erinnerungen wie Merkzeichen gelöscht werden können. Das Meer kann die Gelegenheit geben zu erinnern. Aber es gibt auch ein Meer des Vergessens.

\section{LITERATUR}

Blumenberg, Hans (1979): Schiffbruch mit Zuschauer: Paradigma einer Daseinsmetapher. Frankfurt a.M.

Corbin, Alain (1990): Meereslust. Das Abendland und die Entdeckung der Küste 17501840. Aus dem Franz. v. Grete Osterwald. Berlin. 
Engelbrecht, Johann Andreas (1790): Corpus luris Nautici oder Sammlung aller Seerechte der bekanntesten handeInden Nationen alter und neuer Zeiten: nebst den Assecuranz-Havarey u.a. zu den Seerechten gehörenden Ordnungen. Bd. 1. Lübeck.

Fiske, John (2000): Lesarten des Strandes. In: Ders.: Lesarten des Populären. Aus dem Engl. v. Christina Lutter, Markus Reisenleitner u. Stefan Erdei. Wien, S. 56-95.

Grotius, Hugo (1916): The Freedom of the Seas, or the Right Which Belongs to the Dutch to take part in the East Indian Trade [1608]. Hg. u. eingel. v. James Brown Scott. Aus dem Latein. v. Ralph Van Deman Magoffin. New York; online unter: https://oll. libertyfund.org/titles/552 [Stand: 1.10.2020].

Hansen, Nils (2001): Strandrecht und Strandraub - Bemerkungen zu einem Gewohnheitsrecht an den schleswig-holsteinischen Küsten. In: Kieler Blätter zur Volkskunde Bd. 33, S. 51-78.

Heine, Heinrich (1826): Reisebilder. 1. Theil. Hamburg.

Holm, Christiane (2014): [Art.] "Erinnerungsdinge". In: Susanne Scholz/Ulrike Vedder (Hg.): Handbuch Materielle Kultur. Bedeutungen, Konzepte, Disziplinen. Stuttgart/Weimar, S. 197-201.

Kant, Immanuel (1993): Die Metaphysik der Sitten [1797]. Werkausgabe Bd. VIII. Hg. v. Wilhelm Weischedel. Frankfurt a.M.

König, Gudrun M. (1996): Eine Kulturgeschichte des Spaziergangs. Spuren einer bürgerlichen Praktik 1780-1850. Wien.

Krohn, Tim (2014): Aus dem Leben einer Matratze bester Machart. Roman. Berlin.

Lacan, Jacques (1975): Das Seminar über E.A. Poes Der entwendete Brief. In: Ders.: Schriften I. Aus dem Franz. v. Rodolphe Gasché u.a. Frankfurt a.M., S. 7-61.

Lenz, Siegfried/ Lenz, Liselotte (2011): Kleines Strandgut. Hamburg. Mann, Thomas (1981): Der Zauberberg [1924]. Roman. Frankfurt a.M.

Moser, Christian/Schneider, Helmut J. (2007): Einleitung. Zur Kulturgeschichte des Spaziergangs. In: Axel Gellhaus/Christian Moser/Helmut J. Schneider (Hg.): Kopflandschaften - Landschaftsgänge. Kulturgeschichte und Poetik des Spaziergangs. Köln/Weimar/Wien, S. 9-27.

Neumeyer, Harald (1999): Der Flaneur. Konzeptionen der Moderne. Würzburg. Niehaus, Michael (2003): Das Verhör. Geschichte - Theorie - Fiktion. München.

Ders. (2009): Das Buch der wandernden Dinge. Vom Ring des Polykrates bis zum entwendeten Brief. München

Niitemaa, Vilho (1955): Das Strandrecht in Nordeuropa im Mittelalter. Helsinki 1955.

Rath, Jürgen (2007): ... doch stehlen können sie meisterlich! Über Strandungen, Strandräuber und Strandvögte. Hamburg.

Schelle, Karl Gottlob (1990): Die Spatziergänge oder die Kunst spatzierenzugehen [1802]. Hg. v. Markus Fauser. Nachdruck der Erstausgabe. Zürich/New York.

Schmitt, Carl (1993): Land und Meer. Eine weltgeschichtliche Betrachtung [1942]. Stuttgart.

Simon, Heinrich (1844): Das preußische Staatrecht. 2. Teil. Breslau.

Sommer, Manfred (2002): SammeIn. Ein philosophischer Versuch. Frankfurt a.M. 
Strecker, Werner (1925): Das vormalige Küstengewässer (Strand) und die Rechtsverhältnisse in der Travemünder Bucht. In: Jahrbücher des Vereins für Mecklenburgische Geschichte und Altertumskunde 89, S. 1-228; online unter: http://mvdok. Ibmv.de/mjbrenderer?id=mvdok_document_00003559 [Stand: 1.10.2020].

Tesdorpf, Wilhelm (1887): Gewinnung, Verarbeitung und Handel des Bernsteins in PreuBen von der Ordenszeit bis zur Gegenwart. Jena.

Thumfart, Johannes (2009): 400 Jahre Freiheit der Meere - 400 Jahre Ökonomische Theologie? Ideengeschichtliche Skizze zu Hugo Grotius und Francisco de Vitoria, nach Giorgio Agamben, Carl Schmitt und Max Weber. In: Norman Weiß (Hg.): Hugo Grotius: Mare Liberum. Zur Aktualität eines Klassikertextes. Potsdam, S. 25-32.

Timrott, Jennifer (2015): Strandgut aus Plastik und anderer Meeresmüll. Hamburg. VVVTexel (Hg.; o.J.): Juttersmuseum Flora; online unter: https://www.texel.net/de/veran staltungsuebersicht/kultur/museen/juttersmuseum-flora-257 [Stand: 1.10.2020]. Wagner, Herbert (1997): Die Lex Rhodia de iactu. Marburg.

Weber, Heike (2014): [Art.] "Abfall«. In: Susanne Scholz/Ulrike Vedder (Hg.): Handbuch Materielle Kultur. Bedeutungen, Konzepte, Disziplinen. Stuttgart/Weimar, S. 157160.

Wikipedia (Hg.; 2020a): [Art.] „Flaschenpost«; online unter: https://de.wikipedia.org/ wiki/Flaschenpost [Stand: 1.10.2020].

Wikipedia (Hg.; 2020b): [Art.] „Friendly Floatees«; online unter: https://de.wikipedia. org/wiki/Friendly_Floatees [Stand: 1.10.2020].

Wikipedia (Hg.; 2020c): [Art.] "Plastikmüll in den Ozeanen«; online unter: https:// de.wikipedia.org/wiki/Plastikm\%C3\%BCII_in_den_Ozeanen [Stand: 1.10.2020]. Wolf, Burkhardt (2013): Fortuna di mare. Literatur und Seefahrt. Zürich/Berlin. 